\title{
Petit apologue de nuit
}

acan et Barthes ont connu tous les deux une mort subreptice, comme si, toutes proportions gardées, cette mort n'était pas en rapport avec eux, comme si elle les avait pris tous deux à revers, à la fois par surprise et dans une rhétorique pointilleuse de réalité.

Leur taille semblait les garantir pourtant de ce genre d'accident légèrement obsolète et qui n'arrive plus. Il nous semblait les voir nous parler, à leur front magnétique, à leurs pull-overs tricotés que les fatalités des biologies pouvaient fonctionner pour elles-mêmes, sous des registres sans rapport avec l'esprit et le bon goût.

Et pourtant, Lacan meurt tout de même, innommé, anonyme, inconnu en quelque sorte, sur rendez-vous et sous un nom d'emprunt, à la clinique. Après je ne sais quel abstrait dissentiment, cette palpitation qui nous tient tous a tenu à le disqualifier, lui, le père sévère.

Et Barthes, si doux, lui si artiste, que la stupidité des lois de la gravitation ait semblé agencer tout exprès pour lui cette incompréhensible et insupportable tyrannie mécanique des masses pour l'écraser, comme une chose quelconque. Un camion et pourquoi pas un aérolithe ou encore des formes confuses de structures abstraites et contondantes?

On me dira que Foucault aussi est mort et même, qui l'eût cru, l'inusable Sartre! Mais peut-être quelque chose chez eux pouvait-il laisser présager déjà cette sorte de familiarité à l'absence, une aisance à s'éclipser. Le phénoménologique ou l'existentiel ne méconnaissent rien de ces régions létales, ils vivent au contraire de ce qui les termine. Sartre, j'imagine, a dû rentrer toujours argumentant dans les eaux stygiennes de son enfer meublé Louis-Philippe. Quelque chose même dans la réalité avait dû lui tenir lieu de mort réalisée. Pas d'abstraction surtout, Simone pouvait faire l'affaire pour un adieu sans cérẻmonie. Peut-on se représenter seulement ce géant courbé à l'heure de l'agonie? Je ne peux croire que la mort sans fléchir s'approche de tels êtres. S'imagine-t-on cette ombre notariale se saisissant sans un pli de Foucault! Et dans quel but d'ailleurs, pour quelle sorte d'abstruse logique locale?

Ils étaient tous si bien nés, de cette éternité des demi-dieux, disserts, agrégés, professeurs pour tout dire. Et nous, renvoyés à la 
perpètuité enchantée des potaches, nous avions cru pêle-mêle à cette inébranlable potestas des papas, des oncles, des instituteurs laïcs, et des élus SF10. Toute cette enfance, qui ne passera pas, la vie en somme, végétait paisible et vaguement quiète à l'ombre formidable de nos maitres glorieux.

On ne peut que se demander ce que ces destins avaient tous de si commun pour que, finalement, d'une manière si piètrement identique, ils se soient par mille stratagèmes imprévisibles laissés gagner à cette calamité désuète. La vie nous devrait pourtant cette décence minimale de traiter ceux qui l'ont si brillamment incarnée par une exemption de ses familiarités. Mourir est déjà suffisamment regrettable en soi sans que, dans cette débâcle brouillonne, partent avec elle les meilleurs comme les pires.

One peut se prendre à imaginer que cette tendresse embarrassée qui nous étreignait à les voir passer à la TSF ou sur le gris de nos écrans gaulliens nous garantissait pourtant qu'un ordre immuable en quelque sorte régirait pour toujours la forme innée de nos pensées, de nos rêveries et, qu'à jamais, Roland, Jacques, Jean-Paul ou Michel auraient donné forme à nos générations imprécises, auraient conçu et façonné notre devenir, délié nos méninges, élucidé tout à la fois nos émois estudiantins comme nos progrès séculiers.

Ce siècle d'ailleurs était fabuleux qui voyait ces Pléiades lumineuses, paternelles et bonhommes s'empoigner, qui avec l'inconscient, qui avec la langue, qui avec l'absurde et le politique. Jamais le savoir n'eut si prodigieusement l'air d'une lande aussi rageusement parcourue. Jamais une telle masse de surhommes n'avait encombré à la fois les parvis nationaux. Inoubliable France! Et généreuse, puisqu'à ses morts seuls elle réserve la grandeur.

Ces années sont passées trop vite et cette faculté qu'a le temps de tout jaunir n'a rien épargné. Ce silence excathedra tombé soudain des amphithêâtres (j'allais dire Amphi Geismar ou Bakounine) nous laisse avec ce sentiment diffus d'avoir vu Athènes à Nanterre, entrevu Byzance à Billancourt et vécu au siècle de Périclès.

Sur l'Agora au front de Seine, le ciel s'est entrouvert puis refermé.

Sion Hamou (University of Arizona) 